\title{
LA OPINIÓN DE LOS CONSUMIDORES ESPAÑOLES SOBRE LOS ALIMENTOS TRANSGÉNICOS Y SU SEGURIDAD
}

\author{
THE SPANISH CONSUMERS' OPINION ABOUT GENETICALLY \\ MODIFIED FOOD AND THEIR SAFETY
}

\author{
EMILIO MUÑOZ, MARTA PLAZA, GLORIA PONCE, \\ DAVID SANTOS y OLIVER TODT \\ Departamento de Ciencia, Tecnología y Sociedad, Instituto de Filosofia (CSIC).
}

\begin{abstract}
PALABRAS CLAVE ADICIONALES
Percepción Social, Biotecnología, Usuarios de la

Tecnología, Información, Conocimiento.

\author{
ADDITIONAL KEYWORDS \\ Social Perception, Biotechnology, Users of \\ Technology, Information, Knowledge.
}

RESUMEN. El presente artículo resume los resultados de una encuesta a ciudadanos españoles sobre su percepción de la seguridad alimentaria, especialmente en el área de los productos transgénicos. Los resultados indican que las personas se comportan de dos maneras distintas en el momento de comprar un producto: por un lado, como consumidores de un determinado producto $y$, por el otro, como usuarios (o ciudadanos-usuarios) de tecnología. En ello influye que existe un alto nivel de información general sobre la biotecnología, pero todavía hay poco conocimiento sobre los aspectos específicos de los productos. La tecnología de los alimentos transgénicos se percibe en claves parecidas a los alimentos industriales existentes, lo que influye en las actitudes de compra (mayoritariamente de rechazo). Estas actitudes guardan relación con las respuestas aducidas para justificar el establecimiento de regulaciones (etiquetado) sobre la comercialización de los alimentos modificados genéticamente.

ABSTRACT. This paper presents the results of a study on the public perception of food safety issues and transgenic foods among Spanish citizens. The results show two distinct types of behavior with respect to the acquisition of a product based on a new technology (like biotechnology): the behavior as consumers of a product, and the behavior as citizen and user of this technology. One of the reasons for this schism is the difference between the relatively high level of (general) information and the

\footnotetext{
* Los resultados presentados en este artículo se basan en el "Estudio sobre la sociedad civil y la gobernanza de la ciencia y la tecnología en España", financiado por la Fundación Española para la Ciencia y la Tecnología (FECYT) a través de un convenio entre la Fundación y el CSIC. Asimismo, se ha contado con la ayuda del proyecto BIODIF (BIO 2000-0167-P4-03), financiado por el Plan Nacional de I+D (2000-2003).

E-mail: emiliomz@iesam.csic.es martaplaza@iesam.csic.es gloria.ponce@iesam.csic.es davidsantos@iesam.csic.es todt@iesam.csic.es
}

Revista Internacional de Sociología (RIS)

Tercera Época, No 41, Mayo-Agosto, 2005. pp. 93-108. 
RIS

REVISTA INTERNACIONAL DE SOCIOLOGIA

$\mathrm{N}^{\circ} 41, \mathrm{MAYO}$-AGOSTO, 2005

EMILIO MUÑOZ, MARTA PLAZA, GLORIA PONCE, DAVID SANTOS y OLIVER TODT

low level of (specific) knowledge with respect to this technology. People in Spain perceive transgenic foods as similar to existing industrial foodstuffs. This leads a majority of them to reject purchasing those products. These attitudes are in line with the responses given in relation to the introduction of regulation (labeling) of transgenic foodstuffs.

\section{INTRODUCCIÓN}

El desarrollo científico y tecnológico ha experimentado importantes cambios en las últimas décadas, como consecuencia de la evolución de los contextos sociopolíticos y económicos que ejercen su influencia en esos procesos.

Entre los cambios más relevantes están la transición desde una sociedad industrial a una sociedad de servicios y la emergencia de conceptos como el de "sociedad del riesgo", acuñado por Ulrich Beck (1992) para dar cuenta del tránsito desde la modernización sin más a la "modernización reflexiva", que entraña la inversión de la relación entre la lógica de producción de bienestar y la lógica de producción de riesgo.

Estos cambios han supuesto un giro respecto a la situación en la que los actores del proceso científico y tecnológico deben moverse para alcanzar un satisfactorio desarrollo de sus actividades. La nueva situación abarca tanto la evolución de las políticas y de sus elementos operativos, como la aplicación de los instrumentos para el análisis de la relación entre la producción de conocimiento y sus aplicaciones, así como para el de las interacciones de la ciencia con la tecnología. Este proceso ha conducido a la puesta en práctica de un nuevo contrato social entre la ciencia y la sociedad, en el que la ciencia ya no goza de la consideración de "frontera sin fin", sino que ha perdido una cierta autonomía en función de nuevas demandas sociales.

El contrato social que se estableció en los años cincuenta con las propuestas declarativas de Vannevar Bush reclamaba la protección de los investigadores (su independencia) frente a las presiones políticas, económicas y sociales, y apuntaba que la actividad de los científicos no debía verse restringida por nada que no fuera las propias capacidades de los investigadores. Esta relación entre Estado, política y ciencia condujo a la construcción del mito de la "investigación sin trabas" (Sarewitz, 1996), que se ha venido abajo por la presión de realidades concretas, como han sido los periodos de crisis y recesión económica, o por el debate articulado alrededor de las críticas sociales acerca del modelo idealizado del riesgo que plantean y promueven los científicos.

\section{Evaluación económica y social}

En el ámbito económico, el reconocimiento de que la inversión en investigación y desarrollo (I+D) da lugar a una tasa razonable de retornos financieros, no deja de 
abrir dudas en la línea del conflicto entre el huevo y la gallina, sobre si esa inversión en I+D es realmente motor del bienestar económico o, si por el contrario, es este beneficio el que impulsa la inversión en las actividades de I+D. Hay ejemplos que apuntan tanto en una como en otra dirección, como es el caso de los Estados Unidos o de algunos países desarrollados de Europa, en el primer sentido, frente a la Europa de los quince en su conjunto, o el caso español de modo específico, en la segunda dirección.

Desde el plano de la posición crítica de carácter social, cabe mencionar la exclusión de la reflexividad en la interacción política y social entre expertos y grupos sociales, en relación con los modernos riesgos, a causa de la asunción del realismo como principio de la ciencia. En este contexto, el modelo ideal de sistema de riesgo y las evaluaciones relativas al mismo están basadas en las opiniones de los científicos que se apoyan fundamentalmente en el conocimiento experimental, en los resultados del laboratorio. Este modelo ha sido cuestionado por los científicos sociales, quienes lo acusan de ofrecer un modelo ingenuo de sociedad. En este sentido, algunas orientaciones de la sociología de la ciencia han llevado a cuestionar las asunciones científicas de ese modelo, como podrían ser las visiones constructivistas más fuertes dentro del discurso postmodernista. Lash y Wynne (Beck, 1992) han señalado que la imposición de premisas restrictivas ha supuesto que el debate se haya polarizado, hacia "la distracción realista referente al valor verdadero de las propuestas científicas y la polémica acerca de la pretendida irracionalidad del discurso social anti-científico y tecnológico, con la consiguiente acusación de corrupción de los científicos y de las instituciones reguladoras"1.

Todo este complejo contexto ha conducido a la idea de que la ciencia, el progreso científico y técnico, debe estar sujeto a mayor control social, aumentando sus niveles de "responsabilidad ante la sociedad" ("social accountability").

\section{Evaluación social de las tecnologías}

Esta necesidad reclama la búsqueda de instrumentos para medir y promover la participación social en la evolución del proceso científico y tecnológico y así como las posibles consecuencias del mismo.

Desde una perspectiva técnica, es preciso reconocer que las orientaciones de la política científica no deben situarse en una distinción entre extremos: entre el trabajo que realizan los científicos y lo que se produce o recoge por los no-científicos; entre lo que, en el ámbito de una investigación sin trabas, se juzga sólo por

\footnotetext{
${ }^{1}$ Esta situación ha estado motivada por una serie de ejemplos, negativos generalmente, vinculados a crisis alimentarias o problemas de salud relacionados con el medio ambiente, como, por ejemplo, los casos de las "vacas locas", las dioxinas o los efectos de los herbicidas.
} 
expertos ("pares")y lo que supone una investigación por objetivos en la que políticos y actores sociales intervienen con sus opiniones para decidir lo que se debe hacer. La senda que parece lógico seguir, trata de aumentar el reconocimiento de la ciencia, no por la vía de "ennoblecer" a los científicos y técnicos, sino por la implicación de los actores sociales con el fin de contribuir a la "democratización" de los procesos de decisión en la promoción y el desarrollo de la ciencia y la tecnología.

Este objetivo no es sencillo y ha sido uno de los principios en los que se han asentado los estudios sobre ciencia para poner en práctica la "evaluación de tecnologías". El papel de las ciencias sociales en esta tarea es decisivo aunque no ha dejado de estar sujeto a un profundo debate tanto en términos académicos como funcionales. Desde el plano institucional, la OCDE y la Unión Europea han llevado a cabo estudios, programas e iniciativas para avanzar en esta dirección. El concepto de "Evaluación de Tecnologías" (TA) que hizo fortuna en la década de 1980 y siguió siendo objeto de estudio en la primera mitad de la siguiente década, ha entrado en crisis sobre todo en lo que concierne al valor predictivo de la influencia socio-económica de las tecnologías en el proceso de innovación (véase, como ejemplo, el volumen Social Sciences and Innovation, resultado de un taller organizado por la OCDE en Japón a finales del año 2000).

\section{Percepción pública de la ciencia y la tecnología}

Posteriormente, han ido alcanzando predicamento los estudios sobre opinión pública de la ciencia y la tecnología. Los orígenes de estos trabajos se sitúan en dos movimientos: uno norteamericano ("scientific literacy") y otro británico ("public understanding of science"), que presentan una cierta divergencia en sus propósitos, divergencias que han dado lugar a ciertas dificultades en la aplicación e interpretación de los resultados de esta tecnología de análisis social.

El primero de ellos ha buscado medir el grado de cultura científica o de alfabetización científica, mientras que el segundo, más amplio, persigue valorar la capacidad del público, de la sociedad, para comprender la cienciay, eventualmente, sus aplicaciones. Nuestra más reciente interpretación es que esta estrategia es una forma "nueva" de aproximarse a los ejercicios de "evaluación de tecnologías" en los que se implica a la ciudadanía.

\section{EL CASO DE LA BIOTECNOLOGÍA}

La biotecnología es una tecnología a la que se identifica como "nueva tecnología". Su desarrollo ha estado en el centro del debate social a lo largo de los últimos 25 años. 
En los análisis llevados a cabo en nuestro grupo de trabajo (véase por ejemplo, Muñoz, 1997, 2001) se ha puesto de relieve la complejidad de los temas que han rodeado este debate social, circunstancia que condiciona los estudios sociales sobre esta tecnología (Muñoz, 2002). Se ha señalado que en el debate se entrèmezclan varios factores: deficiencia cognitiva, riesgo, incertidumbre, valores religiosos y morales, intereses y confianza, son algunos de los que emergen.

\section{Las encuestas europeas sobre biotecnología}

La importancia creciente del debate sobre biotecnología ha centrado el interés en las encuestas de opinión sobre la tecnología en cuestión. La Comisión Europea estuvo al frente de este movimiento al promover una política europea en ciencia y tecnología. La introducción de nuevas tecnologías entre las prioridades de la I+D comunitaria en 1980 estuvo enmarcada en un ejercicio de "evaluación de tecnologías", articulado en el programa FAST (Forecast and Assessment of Science and Technology), dirigido por Ricardo Petrella, uno de los pioneros europeos en los estudios sobre ciencia y tecnología. Desde el principio, el programa FAST reconoció la relevancia de los estudios sociales para la introducción de la biotecnología en el Programa Marco de I+D europeo.

\section{Resumen crítico de las encuestas europeas sobre biotecnología (Euro- barómetros)}

En una serie de recientes publicaciones se han analizado los problemas presentes en este tipo de encuestas, tanto desde el punto de vista metodológico como interpretativo (Muñoz, 2002; Pardo y Calvo, 2002; Muñoz, 2004). A modo de resumen se ofrecen los principales puntos resultantes de estos análisis.

En Europa, se han realizado periódicamente por parte de la Comisión Europea, dentro de su colección de Eurobarómetros, una serie de estudios especiales sobre los europeos y la biotecnología. Estos Eurobarómetros, que se han llevado a cabo en los años 1991, 1993, 1996, 1999 y 2002, están basados en la teoría del "déficit cognitivo", contrastada sobre la base de la realización de encuestas con el propósito de valorar la cultura científica de los europeos, con algunos limitados cambios en los últimos años. Estos estudios cuantitativos, según nuestros análisis, han estado orientados a considerar a los ciudadanos como usuarios de tecnología, en un tipo de ejercicio en la línea del "technology assessment", sin hacer hincapié en su condición de consumidores. Existe una gran dificultad para afrontar la complejidad de la biotecnología, tecnología basada en el conocimiento, en rápida evolución y de carácter multisectorial. Los cuestionarios de sus últimas versiones han tratado de afrontar este problema planteando preguntas sobre un 
número limitado de aplicaciones biotecnológicas (alrededor de seis), conectadas en diferentes sectores (agrobiotecnología, farmacobiotecnología, y otros).

Entre los datos más relevantes obtenidos de forma repetida en los Eurobarómetros, merecen ser destacados varios, que exponemos a continuación. En primer lugar, el alto porcentaje de respuestas enmarcadas en la categoría de "No Sabe/No Contesta", que en todas las preguntas suele ser mayor del 30\%, no cambia con el tiempo. En segundo lugar, existe una marcada ambivalencia de los europeos en cuanto a la actitud que afirman tener con respecto a las distintas aplicaciones biotecnológicas: en el caso de las aplicaciones biotecnológicas relacionadas con el tema de salud, la aceptación puede calificarse como media-alta. En tercer lugar, en cuanto a las aplicaciones relacionadas con la agricultura, los encuestados declaran una aceptación media. Y, en cuarto y último lugar, en el caso de la biotecnología aplicada a la obtención de alimentos transgénicos, la aceptación de los europeos se mueve en un intervalo de baja a media aceptación.

En cuanto a la comparación de los resultados de los Eurobarómetros entre países, se comprueba un variado abanico de opiniones dependiendo de las aplicaciones y los países. De forma general, puede observarse que Finlandia, España y Portugal muestran unas actitudes más optimistas con respecto a la biotecnología. En unas posiciones intermedias, se sitúan Holanda, Reino Unido, Suiza, Dinamarca, Irlanda y Bélgica. Las actitudes de Francia e Italia se revelan variables: desde actitudes muy críticas a actitudes más moderadas. Por último, las actitudes de Alemania, Austria, Luxemburgo y Grecia en los últimos tiempos pueden ser consideradas las más críticas.

Si consideramos los distintos grados de aceptación de la modificación genética aplicada a organismos, tenemos, de mayor a menor aceptación, una escala que comienza con los microorganismos, continúa con las plantas, seguida de los animales, hasta llegar al extremo del rechazo en el caso de los seres humanos. Refiriéndonos a las "nuevas aplicaciones biotecnológicas", las aplicaciones más "aceptadas" tienen que ver con la relación entre genética y enfermedad: diagnóstico genético, terapia génica. En posiciones más negativas están los niveles de aceptación de la biotecnología aplicada a la agricultura y, en último lugar, con las opiniones más negativas, se sitúa la biotecnología aplicada a la alimentación.

En nuestro país, se han llevado a cabo algunos estudios recientes de ámbito nacional sobre el tema. Una fuente oficial y neutral de estudios sobre biotecnología y seguridad alimentaria la constituye el Centro de Investigaciones Sociológicas, que ha desarrollado dos estudios monográficos, en los años 1996 y 2001. En dichos estudios se ha encuestado a 2500 españoles sobre los temas monográficos de las "opiniones y actitudes de los españoles ante la biotecnología". En ellos se evidencian intentos de cambiar la tendencia de los cuestionarios hacia la consideración de los encuestados como consumidores, particularmente en agrobiotecnología. 
Por otra parte, la Fundación BBVA, hizo públicos en 2003 los resultados de una encuesta sobre biotecnología, en la cual se profundizaba especialmente en las aplicaciones biotecnológicas de la investigación con embriones y en los alimentos transgénicos.

En general, los resultados de los estudios españoles se corresponden en gran medida con los datos generados por los Eurobarómetros. En España se revela una mayor confianza en la ciencia que en la tecnología. Por otro lado, el porcentaje de encuestados que se coloca en la categoría de "No sabe/No contesta", es también muy alto, con valores que superan el $30 \%$. Además, los niveles de educación y cultura son unas variables con gran potencial explicativo para la comprensión de las actitudes hacia la biotecnología.

\section{ENCUESTA DE ACTITUDES ANTE LA SEGURIDAD ALIMENTARIA Y LOS ALIMENTOS TRANSGÉNICOS}

\section{Temática de la encuesta (comparación con los Eurobarómetros)}

El presente estudio es uno de los primeros realizados en España (para un análisis estadístico con enfoque similar, véase Angulo, Gil y Noomene, 2003) en los que los encuestados son considerados en su doble vertiente de consumidores y usuarios de los alimentos transgénicos, superando la perspectiva tradicional de las encuestas dirigidas a evaluar el grado de conocimiento y de precaución de los ciudadanos sobre los temas, por un lado, de las crisis alimentarias y, por otro, de la biotecnología en general. El estudio, apartándose de la tendencia generalizada hasta momentos recientes, de recabar datos sobre el grado de conocimiento de los ciudadanos sobre el tema de los alimentos transgénicos, se ha focalizado en intentar desentrañar los procesos sociales y las motivaciones individuales que configuran las actitudes y el comportamiento del consumidor.

\section{Metodología}

La encuesta ha sido realizada mediante la metodología CATI (encuesta telefónica asistida por ordenador) cuyo trabajo de campo tuvo lugar en el mes de julio de 2004. El universo de la encuesta lo constituyen los españoles de 18 o más años. La muestra, escogida mediante selección aleatoria a partir de guías telefónicas y ponderada según los datos del censo de sexo y edad, fue de 1.002 entrevistas, lo que supone un error estadístico del $3 \%$ para un nivel de confianza del $95 \%$. La encuesta contó con un pretest en el que se modificaron las preguntas que no se comprendían suficientemente por los entrevistados. 


\section{RIS}

\section{Presentación de los resultados de la encuesta}

Como se puede observar en la Gráfico 1, las tres cuartas partes (75\%) de los ciudadanos entrevistados aseguran conocer la existencia de organismos modificados genéticamente.

\section{Gráfico 1.}

Información sobre la existencia de los alimentos transgénicos (Pregunta: “¿Conocía Ud. la existencia de los alimentos modificados genéticamente?")

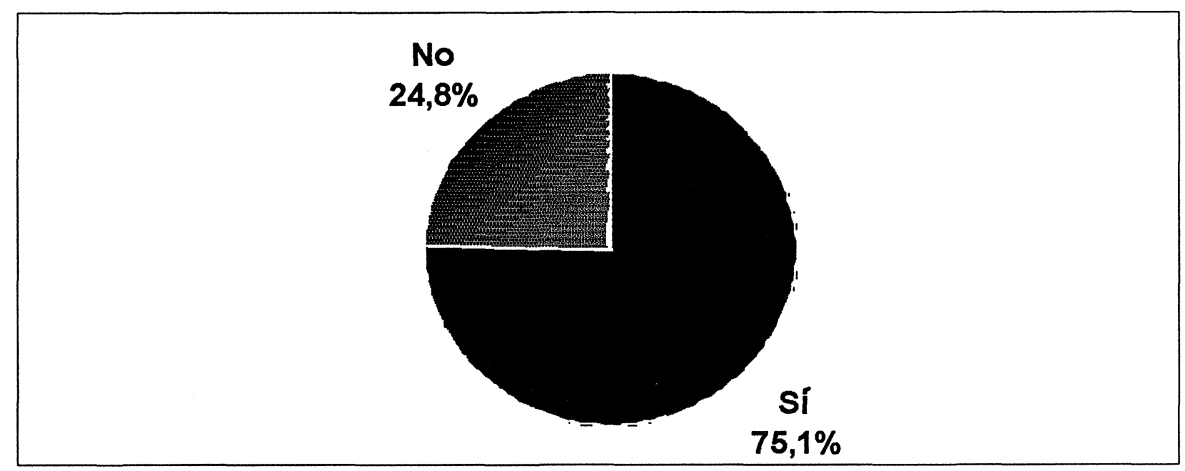

Fuente: Elaboración propia.

\section{Gráfico 2.}

Cualidades de los transgénicos respecto a los alimentos convencionales (Pregunta: "Indique por favor qué ventajas cree que tienen los productos transgénicos respecto a los normales")

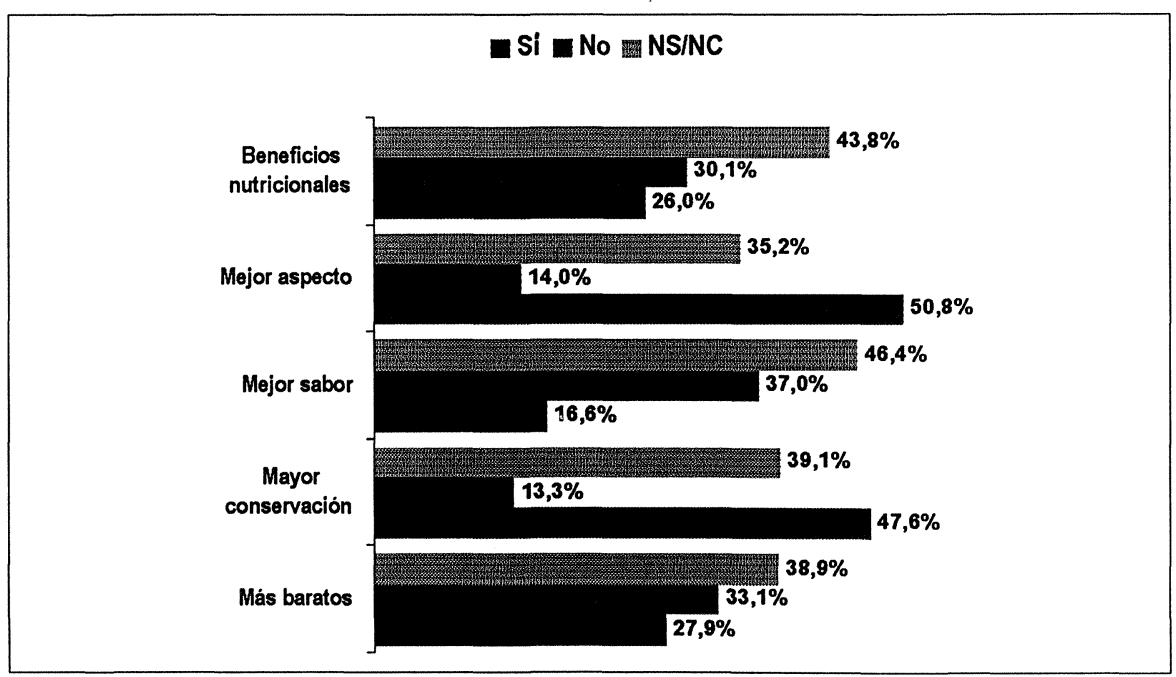

Fuente: Elaboración propia. 
Los entrevistados han valorado algunas cualidades (beneficios nutricionales, mejor aspecto, mejor sabor, mayor conservación y menor precio) de los Organismos Modificados Genéticamente (a partir de ahora OMG) frente a los alimentos convencionales (gráfico 2). Según los resultados obtenidos, parece que la cualidad más reconocida en los $\mathrm{OMG}$ es su mejor aspecto frente a los alimentos convencionales $(50,8 \%)$, siendo la cualidad de una mayor conservación $(47,6 \%)$ la segunda más valorada. Por el contrario, las cualidades de sabor $(16,6 \%)$, beneficios nutricionales $(26 \%)$ y menor precio $(27,9 \%)$, son las menos valoradas, a mucha distancia de las dos anteriores, a juzgar por los porcentajes de respuesta afirmativa en estos casos. Un hecho relevante para las cinco cualidades estudiadas es el alto porcentaje de respuestas "No sabe/ No contesta", situándose entre el $50,8 \%$ y el $35,2 \%$. Es un indicador sobre el alto desconocimiento generalizado que los entrevistados tienen acerca de las propiedades de los OMG.

\section{Gráfico 3.}

Intención de compra de OMG

(Pregunta: “¿Compraría Ud. un producto transgénico?”)

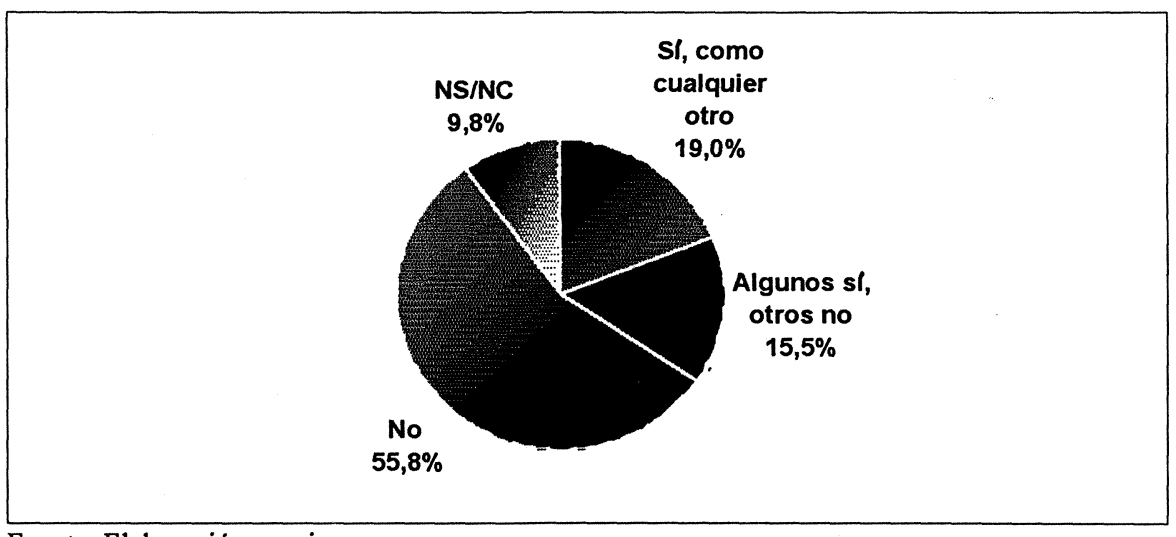

Fuente: Elaboración propia.

Sobre la intención de compra de OMG (gráfico 3), la opinión es desfavorable en un 55,8\% de los casos, siendo en este caso sólo del 9,8\% el porcentaje de indecisos. Sólo el 19\% se muestra firme en la decisión de comprar OMG como cualquier otro tipo de alimento. 


\section{RIS}

Gráfico 4.

Opinión de los consumidores respecto al establecimiento de la legislación sobre etiquetado de los alimentos transgénicos (\% de respuestas afirmativas)

(Pregunta: "Indique las razones por las que cree que se ha establecido una legislación que obliga a indicar en la etiqueta de los alimentos el contenido de productos transgénicos")

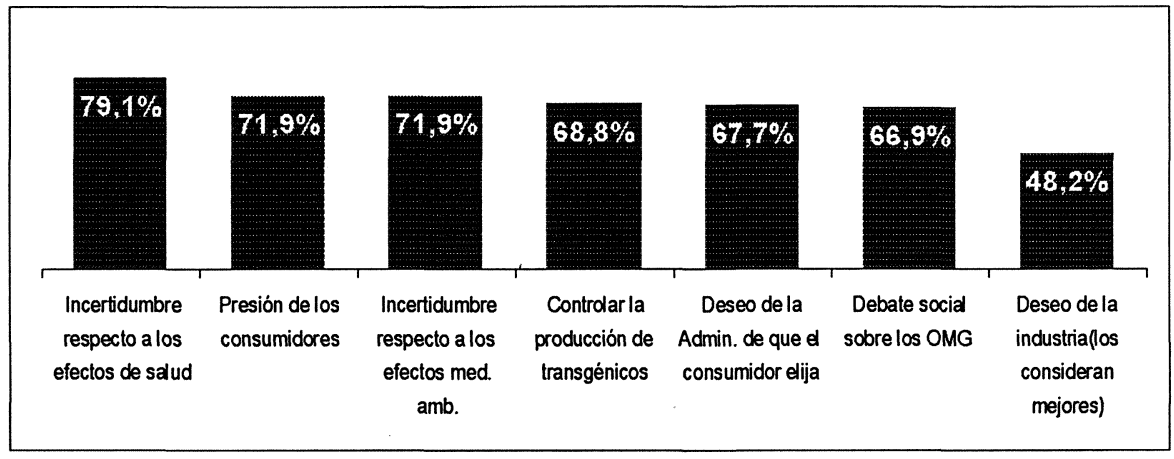

Fuente: Elaboración propia.

Esta pregunta (véase gráfico 4) hace referencia a los motivos por los que los entrevistados creen que se ha establecido la legislación sobre el etiquetado de los OMG. El motivo con más relevancia para los ciudadanos ha sido la incertidumbre que suscitan los OMG respecto a los efectos sobre la salud, con un $79,1 \%$. A continuación existen cinco motivos valorados con similares porcentajes, entre el $71,9 \%$ y el $66,9 \%$. A cierta distancia se encuentra el motivo que hace referencia a los deseos de la industria, que tiene interés en diferenciarlos porque los considera mejores que los alimentos convencionales. Este argumento está respaldado por un $48,2 \%$ de los entrevistados.

En el gráfico 5 se presentan los resultados de la pregunta sobre los motivos que han condicionado la autorización de la venta de los OMG, pudiendo elegir el entrevistado dos causas influyentes según su opinión. Las presiones de determinadas industrias sobre las autoridades es el motivo más señalado, con el $29 \%$ de respuestas afirmativas. En un segundo nivel, se encuentran las presiones internacionales y los informes científicos favorables, con un $20 \%$ y $18 \%$, respectivamente. Un tercer grupo lo forman las peticiones de grupos ecologistas y las peticiones de los consumidores, con un $12 \%$ y $11 \%$. A mucha distancia, con porcentajes alrededor del $1 \%$ o incluso menores, se encuentran los intereses económicos, los intereses políticos, los beneficios para la salud y otras razones. 


\section{Gráfico 5 .}

Evaluación de la autorización de la venta de los organismos modificados genéticamente (OMG)

(Pregunta: "Indique por favor dos motivos por los que Ud. cree que se ha producido esta autorización")

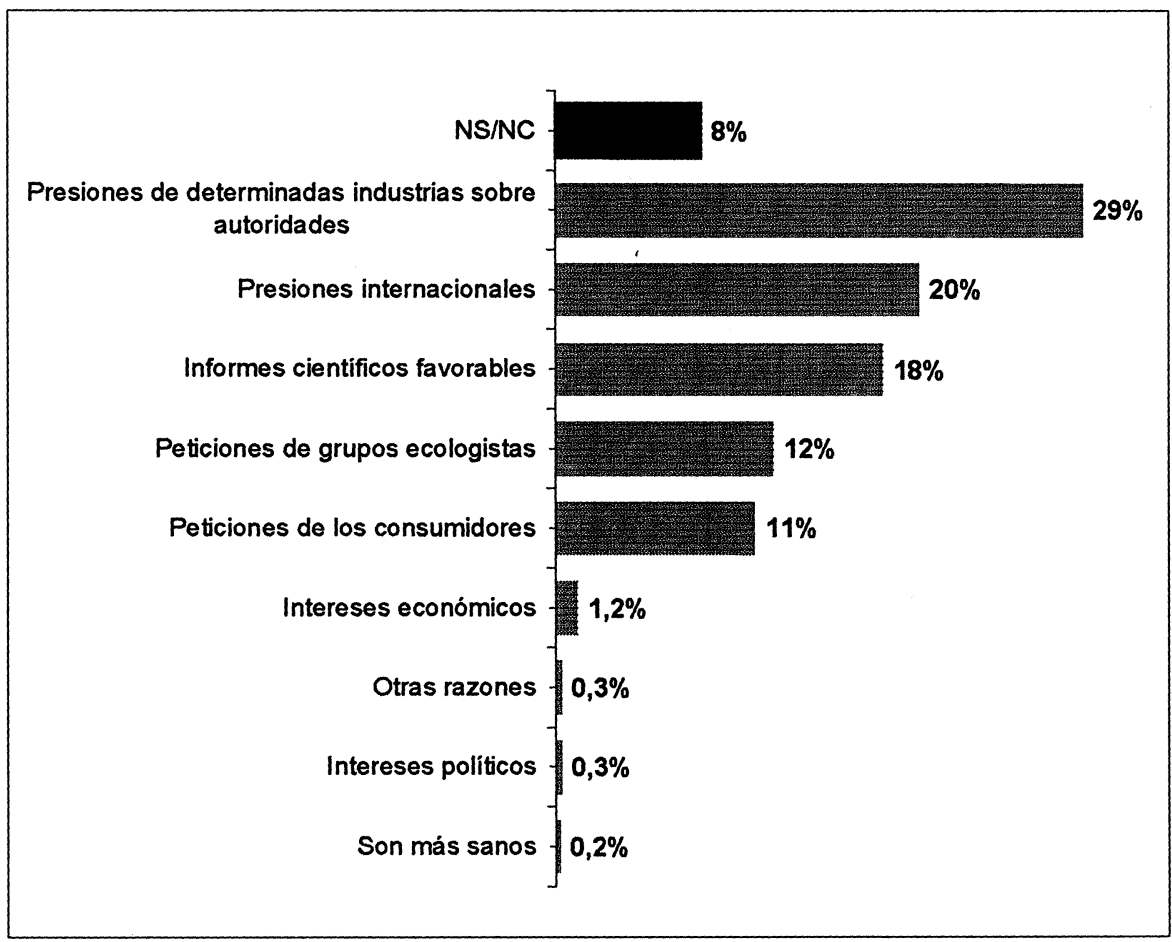

Fuente: Elaboración propia.

\section{ANÁLISIS DE LOS DATOS DE LA ENCUESTA}

\section{Consumidores y usuarios: diferentes formas de relación con la tecnología}

El análisis de los porcentajes de "No sabe/ No contesta" revela la complejidad de la percepción que las personas puedan tener de una tecnología. Pueden actuar en roles distintos (e incluso contradictorios) cuya existencia confirman los datos de la encuesta. Se pueden diferenciar dos tipos básicos de relación con una determinada tecnología o un producto técnico: 
RIS

REVISTA INTERNACIONAL DE SOCIOLOGIA

№ 41, MAYO-AG0ST0, 2005

EMILIO MUÑNOZ, MARTA PLAZA, GLORIA PONCE, DAVID SANTOS Y OLIVER TODT

1) Comportamiento como "consumidor".

La persona debe llevar a cabo un acto de compra que constituye una decisión dirigida al mercado. Esa decisión de compra es de una naturaleza inmediata. Sus motivos pueden ser múltiples, teniendo los motivos económicos (en relación tanto al precio que el consumidor tiene que pagar para el producto como a su influencia sobre la estructura y la conformación de los mercados y las estructuras económicas en cuestión) generalmente un papel muy destacado. Para tomar la decisión de compra, el consumidor tiende a no recurrir a sus conocimientos objetivos en todo detalle ni a una reflexión exhaustiva de todas las implicaciones y características de la tecnología. En cambio, la decisión tiende a basarse sobre una apreciación sintética y general del producto (necesidad, utilidad, experiencia anterior, etc.) sobre cuya compra tiene que decidir.

Estos resultados revelan una clara conciencia de consumidor, que, en general, tiene las ideas claras en cuanto a su intención de compra o no compra; diversos estudios sobre consumidores y alimentos transgénicos han dado a conocer que para poder formarse una decisión, los consumidores demandan información, tanto en el etiquetado, como de los poderes públicos. Tras varios años de debate, en general, los españoles están informados sobre la existencia de los alimentos transgénicos, aunque no llegan a conocer, lo que podríamos llamar desde un punto de vista "científico" en profundidad todas sus implicaciones. Como se puede observar, esa fosa entre información y conocimiento, revelada por la encuesta, tiene importantes implicaciones para la percepción pública de la biotecnología.

2) Comportamiento como usuario o, más precisamente, como ciudadano-usuario.

En ese caso, no se trata de tomar una decisión con implicaciones directas e inmediatas (como la compra de un producto). Se trata de una opinión o percepción (sobre la tecnología o el producto) que, en determinados casos, podría también implicar una decisión, pero con un carácter marcadamente distinto: una decisión de apoyar o rechazar una tecnología en ámbitos como, por ejemplo, el debate público o la legislación (regulación, políticas públicas).

La opinión, la percepción o, en su caso, la decisión implícita se dirige al sistema sociopolítico en general, y está relacionada con la aceptación o el rechazo general de trayectorias tecnológicas y su regulación (evaluación de tecnologías). El usuario, para formar su opinión o percepción, tiende a apoyarse en sus conocimientos objetivos (sobre determinadas características, efectos o procesos en relación con la tecnología o el producto) de manera detallada y reflexionada. Las motivaciones pueden ser múltiples (éticas, socioeconómicas, sociopolíticas, científicas, de intereses, etc.), teniendo la dimensión económica en este caso un 
peso reducido, mientras dominan consideraciones con implicación para el conjunto de la sociedad (dirección del desarrollo tecnológico y sus efectos sobre la sociedad y el individuo, crítica general hacia determinados aspectos del sistema socioeconómico, político o tecnológico, efectos sobre la calidad de vida, implicaciones éticas o religiosas, etc.).

Esos dos papeles diferentes que las personas pueden asumir al formarse una percepción sobre esta cuestión se ven en el gráfico 6 , sobre la base de las importantes diferencias entre los porcentajes de "No sabe/ No contesta" por los mismos encuestados en el mismo proceso de consulta.

En la primera pregunta, que mide la inclinación hacia la compra (o no compra) de un alimento transgénico, hay menos de un $10 \%$ de respuestas "No sabe/ No contesta". En la segunda y tercera pregunta, que miden la percepción sobre los efectos generales de los alimentos transgénicos sobre el medio ambiente y la salud, respectivamente, ese porcentaje sube a un $31 \%$ y un 33\%, respectivamente. $\mathrm{Y}$ en la cuarta pregunta, que mide la percepción en relación con determinadas características muy concretas y detalladas de los alimentos transgénicos (como su sabor, apariencia o conservación), encontramos niveles de respuestas de "No sabe/ No contesta" aún más altos, entre el $35 \%$ y el $46 \%$, con una media de un $40 \%$ de las cinco preguntas parciales.

En la primera pregunta en la que se pide una decisión de compra, la gran mayoría (más del $90 \%$ ) de los encuestados tiene una opinión respecto a comprar o no comprar. En las restantes preguntas, en las que se pide opiniones sobre efectos y características de los alimentos transgénicos, entre un tercio y casi la mitad de las personas consideran que no están en posición de dar una respuesta. Además, el nivel de "No sabe/ No contesta" es marcadamente más alto en la pregunta que pide opiniones sobre características muy concretas de esa tecnología que en las preguntas sobre sus efectos generales sobre la salud y el medio ambiente.

En la primera pregunta, las personas asumen un papel de "consumidor" y son capaces de asumir una decisión basándose en una valoración sintética y general del producto (por ejemplo, su percibida utilidad inmediata o un nivel de precaución deseado). De hecho, se puede suponer que un porcentaje significativo de personas basan su decisión de comprar (o, en este caso, más probablemente de no comprar) en un nivel deseado de precaución, porque mientras el $90 \%$ de los encuestados opina sobre la compra de un alimento transgénico, sólo el $75 \%$ dice estar informado de su existencia. En las otras tres preguntas, las personas asumen el papel de "usuarios". Al dar su respuesta, tienden a hacer recurso a su conocimiento sobre efectos o características de los alimentos transgénicos. Pero, como otras encuestas demuestran (Gaskell y Bauer, 2001), el nivel de conocimiento sobre las características concretas y detalladas de esa tecnología (que implican también conocimientos científicos-técnicos) es aún muy bajo en la población 
RIS

REVISTA INTERNACIONAL DE SOCIOLOGIA

$\mathrm{N}^{\circ}$ 41, MAYO-AGOST0, 2005

EMILIO MUÑOZ, MARTA PLAZA, GLORIA PONCE, DAVID SANTOS y OLIVER TODT

española (como también, aunque menos pronunciado, en la europea). De hecho, se ve claramente cómo el nivel de "No sabe/ No contesta" aumenta cuando se hacen preguntas sobre aspectos más específicos (cuarta pregunta, comparada con la tercera). Otro factor que contribuye es la falta de experiencia práctica de los ciudadanos, dada la práctica imposibilidad actual de adquirir alimentos transgénicos (a menos que sea como ingrediente secundario de algunos alimentos procesados) en la Unión Europea (el proceso de autorización europea arrancó en mayo de 2004, después de seis años de moratoria implícita).

\section{CONCLUSIONES}

La percepción general que las personas tienen de los alimentos transgénicos es de un tipo de alimentos que reproducirán en grandes rasgos las características de los alimentos industriales que actualmente dominan los mercados alimenticios en los países industrializados. La pregunta sobre las características específicas (sabor, valor nutricional, etc.) de los transgénicos con respecto a los alimentos existentes (ver gráfico 2), aparte de mostrar índices muy altos de "No sabe/ No contesta" (entre $35 \%$ y $46 \%$ ), indica que prácticamente la mitad de los encuestados (un $51 \%$ y un $48 \%$, respectivamente) está convencido de que los transgénicos tienen "mejor aspecto" y "un mayor periodo de conservación" (sólo un 14\% y un 13\%, respectivamente, considera que no es así). Pero sólo un $26 \%$ supone que tienen "mayores beneficios nutricionales" (un 30\% supone que no) y sólo un $17 \%$ supone que tienen "mejor sabor" (un $37 \%$ dice que no es así).

En otras palabras, los encuestados perciben que los alimentos transgénicos reproducen principalmente las características de los alimentos industriales existentes (buen aspecto, buena conservación, reducido sabor, etc.). Además, las características de los transgénicos que la mitad da por supuestas son precisamente ventajas principalmente para los vendedores (el buen aspecto es un argumento en el momento de compra, la mayor conservación permite tener los alimentos durante más tiempo en el almacén o la tienda), mientras las características que sólo una minoría da por supuesto serían beneficios más directos para el consumidor, pero que no son comprobables en el momento de compra (sabor), o sólo con métodos dificilmente accesibles a los consumidores normales (valor nutricional comparativo). En otras palabras, la percepción de las personas es no sólo que los alimentos transgénicos no contribuirán a mejorar significativamente el tipo de oferta alimenticia actual, sino que sus beneficios más probables serán, en primer lugar, para los productores (y no para los consumidores). Esa situación se ve complementada por la percepción respecto al precio: sólo un $28 \%$ supone que los transgénicos serán más baratos (lo que constituiría una clara ventaja para los 
consumidores), mientras que un $33 \%$ supone que no. La falta de ventaja percibida para el consumidor se traduce en un rechazo mayoritario de intención de compra de esos alimentos (gráfico 3).

Los datos indican la existencia de una desconfianza hacia los procesos de autorización de los productos modificados genéticamente. El gráfico 5 muestra que una gran mayoría (alrededor de la mitad de los encuestados) supone que fueron las presiones de "determinadas industrias" o "internacionales" sobre las autoridades europeas las que llevaron a la reciente autorización de los productos transgénicos, después de varios años de moratoria. En cambio, sólo un $18 \%$ supone que los "informes científicos favorables" tuvieron un papel importante. La desconfianza hacia el proceso de toma de decisiones y su independencia respecto a la influencia de determinados grupos es un tema interesante en relación con la eficiencia del discurso de "precaución" que abandera la Comisión Europea.

El hecho de haber cambiado la matriz teórica en el diseño del cuestionario acompañado por su simplificación ha permitido dilucidar y caracterizar algunos procesos en el comportamiento de las personas como consumidores y de apuntar diferencias respecto a los actitudes como usuarios. Aun así, sería necesario hacer encuestas más detalladas con el fin de concretar comportamientos.

Con esta aproximación experimental se ha podido establecer un posible camino para distinguir entre los efectos de poseer información y de ostentar conocimiento. En cualquier caso, es evidente que estamos en el principio de una nueva orientación que va a requerir de procesos de consulta detallados para profundizar en el análisis y la interpretación de estos comportamientos

\section{REFERENCIAS BIBLIOGRÁFICAS}

ANGULO, A.M., J.M. GIL y R. NOOMENE (2003), "Consumers' attitudes and buying intentions towards GM food in Spain", $7^{\text {th }}$ ICABR International Conference on Public Goods and Public Policy for Agricultural Biotechnology, Junio 29-Julio 3, Ravello (Italia).

BECK, U. (1992), Risk Society. Towards a New Modernity (traducido por M. Ritter), Londres, Thousand Oaks, Sage Publications, New Delhi.

BUSH, V. (1960), Science, the Endless Frontier (reprint), Washington, D.C., National Science Foundation.

DURANT, J., M.W. BAUER y G. GASKELL (eds.), (1998), Biotechnology in the Public Sphere. A European Sourcebook, Londres, Science Museum.

GASKELL, G. y M. BAUER (eds.) (2001), Biotechnology 1996-2000, the years of controversy, Londres, Science Museum. 


\section{RIS}

LASH, S. y B. WYNNE (1992), "Introduction", en U. Beck, Risk Society. Towards a New Modernity, pp. 1-8, London, Thousand Oaks, New Delhi, Sage Publications

MUÑOZ, E. (1997), Biotecnología, Industria y Sociedad: el caso español, Madrid, Fundación CEFI.

(2001), Biotecnología y Sociedad: encuentros y desencuentros, Madrid, Cambridge University Press, OEI.

(2002), "La cultura científica, la percepción pública y el caso de la biotecnología”, Documento de trabajo 02-07, Madrid, [http://www.iesam.csic.es/doctrab.htm].

(2002), "New socio-political environments and the dynamics of European public research systems" Documento de trabajo 02-20, Madrid, [http://www.iesam.csic.es/doctrab.htm].

(2003), "Problems in the analysis of the public's perception of Biotechnology: Europe and its contradictions", Madrid, Documento de trabajo 03-03, [http://www.iesam.csic.es/doctrab.htm].

(2004), "Los problemas en el análisis de la percepción pública de la biotecnología: Europa y sus contradicciones", en F. J. Rubia Vila (dir.), I. Fuentes Julián y S. Casado de Otaola (coords.), Percepción social de la ciencia, pp. 127-166, Madrid, Academia Europea de Ciencias y Artes, UNED Ediciones.

OECD (2001), "Social Sciences and Innovation", Information Society.

PARDO, R. y F. CALVO (2002), "Attitudes towards science among the European public: a methodological analysis", Public Understanding of Science, pp.155-195.

SAREWITZ, D. (1996), Frontiers of Illusion. Science, Technology and the Politics of Progress, Philadelphia, Temple University Press. 